\title{
A New Weibull-Pareto Distribution: Properties and Applications
}

\author{
M. H. Tahir, Gauss M. Cordeiro, Ayman Alzaatreh, M. Mansoor, M. Zubair \\ Department of Mathematics
}

\begin{abstract}
Many distributions have been used as lifetime models. In this article, we propose a new threeparameter Weibull-Pareto distribution, which can produce the most important hazard rate shapes, namely, constant, increasing, decreasing, bathtub, and upsidedown bathtub. Various structural properties of the new distribution are derived including explicit expressions for the moments and incomplete moments, Bonferroni and Lorenz curves, mean deviations, mean residual life, mean waiting time, and generating and quantile functions. The Rényi and q entropies are also derived. We obtain the density function of the order statistics and their moments. The model parameters are estimated by maximum likelihood and the observed information matrix is determined. The usefulness of the new model is illustrated by means of two real datasets on Wheaton river flood and bladder cancer. In the two applications, the new model provides better fits than the KumaraswamyPareto, beta-exponentiated Pareto, beta-Pareto, exponentiated Pareto, and Pareto models.
\end{abstract}

Original language English

Pages (from-to) 3548-3567

Number of pages 20

Journal $\quad$ Communications in Statistics: Simulation and Computation

Volume $\quad 45$

Issue number $\quad 10$

State $\quad$ Published - Nov 252016

Tahir, M. H., Cordeiro, G. M., Alzaatreh, A., Mansoor, M., \& Zubair, M. (2016). A New WeibullPareto Distribution: Properties and Applications. Communications in Statistics: Simulation and Computation, 45(10), 3548-3567. DOI: 10.1080/03610918.2014.948190 